\title{
Aburrimiento y disfrute en clase de Lengua española en secundaria: predictores motivacionales y efectos sobre el rendimiento
}

\author{
Antonio González ${ }^{1 *}$, Verónica Paoloni² y Cristina Rinaudo ${ }^{2}$ \\ ${ }^{1}$ Universidad de Vigo. España. \\ ${ }^{2}$ Universidad Nacional de Río Cuarto. Córdoba, Argentina
}

\begin{abstract}
Resumen: Este trabajo intenta analizar, mediante un modelo de ecuaciones estructurales, las relaciones entre motivación autodeterminada, aburrimiento, disfrute y rendimiento en clase de Lengua. La muestra estuvo compuesta por 642 estudiantes de secundaria españoles, con edades comprendidas entre 12 y 18 años, que fueron evaluados en tres momentos sucesivos. Los resultados mostraron que la motivación intrínseca y la regulación identificada predijeron positivamente el disfrute y el rendimiento, y negativamente el aburrimiento; amotivación y regulación externa predijeron positivamente el aburrimiento y negativamente el disfrute y el rendimiento; la regulación introyectada mostró unos resultados menos concluyentes; aburrimiento y disfrute predijeron el rendimiento y mediaron las relaciones entre motivación autodeterminada y rendimiento. Se discuten los resultados y la importancia de la autodeterminación y las emociones académicas en el aula.

Palabras clave: Autodeterminación; motivación; emociones académicas; resultados académicos; modelos estructurales.
\end{abstract}

\section{Introducción}

En nuestras aulas, especialmente a partir de secundaria, no resulta extraño ver estudiantes que dormitan, bostezan, miran el reloj o simplemente se distraen mientras esperan que concluya una sesión de clase que les parece interminable. Por fortuna, en otros muchos casos un timbre sorprende a los alumnos anunciándoles el final de una clase que concluyó mucho antes de lo deseado. Éstos son sólo dos ejemplos que apoyan a diferentes autores (Ainley y Ainley, 2011; Meyer y Turner, 2007; Pekrun, Frenzel, Goetz y Perry, 2007) cuando afirman que las emociones influyen de manera importante sobre el aprovechamiento académico. También lo expresan dos informes recientes sobre la educación en España: así, Fernández, Mena y Riviere (2010) aseguran que el aburrimiento en las clases representa uno de los principales procesos que conduce al fracaso y al abandono escolar en secundaria; por su parte, el último informe PISA (Ministerio de Educación, 2010) afirma que el disfrute con la lectura es un fuerte predictor del rendimiento en comprensión lectora. Un condicionante decisivo de estas emociones es la motivación académica, siendo el de la autodeterminación uno de los modelos más investigados (Deci y Ryan, 2000; Ryan y Deci, 2002). A su vez, emociones académicas y motivación ejercen una influencia decisiva sobre el rendimiento (Pekrun, 2009; Ryan y Deci, 2009).

* Dirección para correspondencia [Correspondence address]: Antonio González. Área de Psicología Básica. Universidad de Vigo. Facultad de Ciencias de la Educación. As Lagoas. 32004. Ourense (España).E-mail: aglez@uvigo.es
Title: Boredom and enjoyment in the Spanish language classroom in secondary education: Motivational predictors and effects on performance Abstract: The aim of this study was to undertake a structural equation modelling analysis of the relationship between self-determined motivation, boredom, enjoyment, and performance in the Spanish language classroom. The sample consisted of 642 Spanish secondary education students aged 12 to 18 years, who were assessed at 3 successive intervals. The results reveal that intrinsic motivation and identified regulation positively predicted enjoyment and performance, and negatively boredom; amotivation and external regulation positively predicted boredom, and negatively enjoyment and performance; introjected regulation, however, did not yield conclusive results; boredom and enjoyment predicted performance and mediated the relationships between self-determined motivation and performance. The results are discussed in terms of the significance of self-determination and emotions in academic settings.

Key words: Self-determination; motivation; academic emotions; academic achievement; structural models.

\section{Motivación autodeterminada}

Los autores de la teoría de la autodeterminación diferencian múltiples modalidades de motivación, ordenadas en un continuo de menor a mayor grado de autodeterminación, desde la amotivación hasta la motivación intrínseca (Deci y Ryan, 2000; Ryan y Deci, 2002, 2009). El alumno con amotivación o desmotivación carece de intención de actuar, por lo que su conducta es no-autodeterminada. La motivación extrínseca se define como cualquier situación en la que la razón para la actuación es alguna consecuencia separable de ella, y puede ser de distintos tipos: regulación externa, si las conductas se realizan para satisfacer una demanda exterior; regulación introyectada, cuando las acciones se llevan a cabo bajo un sentimiento de presión, con el fin de evitar la sensación de culpa o ansiedad, o para aumentar la autoestima; regulación identificada, si la persona reconoce y acepta el valor implícito de una conducta y la ejecuta aunque no le resulte agradable; y regulación integrada, cuando la identificación se ha asimilado dentro del propio yo. Son conductas intrínsecamente motivadas aquellas que los sujetos consideran interesantes por sí mismas y que desean realizar en ausencia de consecuencias porque satisfacen sus necesidades personales de autonomía y competencia. La regulación externa y la introyectada se consideran formas de motivación controlada, mientras que la regulación identificada, la integrada y la motivación intrínseca son modalidades de motivación autónoma.

La investigación previa indica que variables como esfuerzo, persistencia, utilización de estrategias y rendimiento académico correlacionan positivamente con la motivación intrínseca y la regulación identificada, y negativamente con la amotivación y la regulación externa; respecto a la regulación 
introyectada, los datos no son tan claros (Assor, Vansteenkiste y Kaplan, 2009; Boiché, Sarrazin, Grouzet, Pelletier y Chanal, 2008; Deci y Ryan, 2000; Liu, Wang, Tan, Koh y Ee, 2009; Ntoumanis, 2002; Roth, Assor, Niemiec, Ryan y Deci, 2009; Ryan y Connell, 1989; Ryan y Deci, 2002, 2009). La regulación integrada apenas se ha investigado (GonzálezCutre, Sicilia y Fernández, 2010).

\section{Emociones académicas: disfrute y aburrimiento}

Las emociones suelen concebirse como procesos psicológicos complejos, con componentes afectivos, cognitivos, motivacionales y expresivos, que se activan como reacción ante objetos, situaciones o eventos significativos (Meyer y Turner, 2007; Pekrun, 2009). Para referirse a las emociones académicas, Pekrun et al. (2007; Pekrun, 2006, 2009) hablan de emociones de logro y las consideran directamente asociadas a actividades y resultados académicos evaluados mediante criterios de calidad. Para clasificarlas, utilizan varias dimensiones, como la valencia (positiva o negativa) y la activación o no de la conducta.

Disfrute y aburrimiento son dos emociones frecuentemente estudiadas en el contexto académico. Así, Ainley y Ainley (2011), Frenzel, Goetz, Lüdke, Pekrun y Sutton (2009) y Goetz, Frenzel, Hall y Pekrun (2008) se centraron en la emoción de disfrute. Por su parte, Acee et al. (2010), Daschmann, Goetz y Stupnisky (2011), Nett, Goetz y Daniels (2010), Nett, Goetz y Hall (2011) y Pekrun, Goetz, Daniels, Stupnisky y Perry (2010) tuvieron en el aburrimiento su foco de análisis. Otros trabajos estudiaron el disfrute y el aburrimiento junto a otras emociones académicas (Daniels et al., 2009; Dettmers et al., 2011; Goetz, Frenzel, Pekrun, Hall y Lüdtke, 2007; Goetz, Hall, Frenzel y Pekrun, 2006; Pekrun, Goetz, Frenzel, Barchfeld y Perry, 2011).

El disfrute es una emoción agradable, activadora de la conducta, que surge cuando una actividad concreta se considera controlable y es valorada positivamente; representa un componente fundamental del interés personal y del valor subjetivo de la tarea (Ainley y Ainley, 2011; Frenzel et al., 2009; Frenzel, Pekrun y Goetz, 2007; Goetz et al., 2006. 2007, 2008). Estas investigaciones, muchas de ellas con alumnos de secundaria, constataron que el rendimiento académico en distintas materias (matemáticas, física, ciencias naturales, inglés, música, alemán o latín) correlacionó intensa y positivamente con el disfrute.

Por su parte, el aburrimiento es una emoción desagradable y desactivadora de la conducta (Acee et al., 2010; Net et al., 2010, 2011; Pekrun, 2006, 2009; Pekrun et al., 2010). El estudiante la experimenta cuando considera escasamente controlable una actividad poco valorada. El aburrimiento provoca la sensación de que el tiempo no pasa, por lo que se desea escapar de esa situación, y se caracteriza por posturas o gestos que denotan falta de ilusión, de interés y de valoración de la actividad. Su relación con el rendimiento académico en diversas asignaturas fue no significativa (Frenzel et al., 2007; Goetz et al., 2007) o negativa (Acee et al., 2010; Nett et al., 2010, 2011; Pekrun, 2006, 2009; Pekrun et al., 2010, 2011).

\section{Relaciones entre autodeterminación y emociones}

Algunos trabajos han abordado los nexos entre motivación autodeterminada y emociones. Así, el disfrute correlacionó negativamente con la amotivación y la regulación externa, y positivamente con la regulación introyectada, la identificada y la motivación intrínseca en clase de educación física (Ntoumanis, 2002) y en la realización de un trabajo grupal extenso (Liu et al., 2009); asimismo en clase de educación física, Wang y Liu (2007) encontraron idénticas relaciones, salvo que la regulación introyectada correlacionó negativamente con el disfrute; en un contexto próximo, el deportivo, Núñez, León, González y Martín-Albo (2011) constataron que diferentes modalidades de motivación intrínseca predijeron positivamente el bienestar personal. Por su parte, el aburrimiento en educación física correlacionó positivamente con la amotivación y la regulación externa (Ntoumanis, 2002; Sas-Nowosielski, 2008); su correlación con la regulación identificada y la motivación intrínseca fue intensa y negativa; la correlación entre aburrimiento y regulación introyectada fue moderada negativa (Ntoumanis, 2002) o no significativa (Sas-Nowosielski, 2008). En universitarios, el aburrimiento en clase correlacionó negativamente con la motivación intrínseca (Pekrun et al., 2010, 2011).

En este trabajo, todas las variables están referidas a las clases de la asignatura de Lengua española, atendiendo a la recomendación de Vallerand (1997) de evaluar la motivation y sus resultados en el mismo nivel de generalidad. Además, siguiendo las indicaciones de Wu y Zumbo (2008), se han evaluado las variables en momentos sucesivos a lo largo del curso, con el fin de establecer más adecuadamente el modelo de mediación en el que la observación o la medida de la variable independiente precede en el tiempo a la observación de la variable dependiente.

Como hemos visto, son escasas las investigaciones que han abordado las relaciones entre motivación autodeterminada y disfrute o aburrimiento en clases de secundaria, más allá de evaluar el bienestar/malestar académicos o la satisfacción/insatisfacción generales. Por tanto, el principal objetivo del presente trabajo es descubrir los nexos entre diferentes modalidades de motivación autodeterminada (amotivación, regulación externa, introyectada e identificada, y motivación intrínseca), aburrimiento y disfrute en clase, y rendimiento en la asignatura de Lengua. La teoría de la autodeterminación propone que la motivación intrínseca y la regulación identificada se asociarán positivamente al rendimiento y a emociones agradables, y negativamente a las desagradables; un patrón inverso de relaciones es el que se pronostica para la desmotivación y la regulación externa; en cuanto a la regulación introyectada, las previsiones no son tan precisas (Boiché et al., 2008; Deci y Ryan, 2000; Ryan y Deci, 2002, 2009). En esta misma dirección apuntan los resultados de las escasas investigaciones empíricas que estudiaron motivación 
autodeterminada y emociones académicas específicas, sobre todo en educación física. Partiendo de estos antecedentes, planteamos el modelo de relaciones que se sintetiza en la Figura 1.

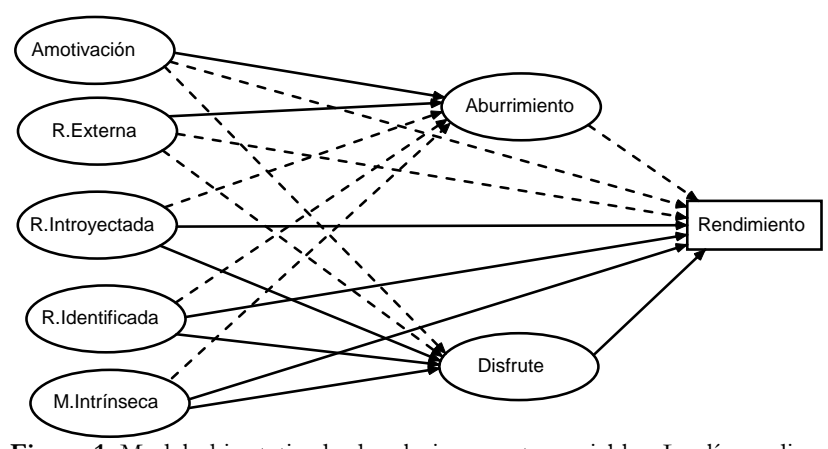

Figura 1. Modelo hipotetizado de relaciones entre variables. Las líneas discontinuas indican nexos negativos.

Concretamente, queremos comprobar si (a) las modalidades de motivación autodeterminada predicen de forma diferencial (según su grado de autodeterminación) el aburrimiento, el disfrute y el rendimiento académico; si (b) aburrimiento y disfrute son predictores significativos del rendimiento; y si (c) disfrute y aburrimiento median las relaciones entre motivación autodeterminada y rendimiento.

\section{Método}

\section{Participantes y procedimiento}

Formaron la muestra 642 estudiantes ( $51 \%$ chicas) con edades comprendidas entre 12 y 18 años $(M=15.2$; $D T=$ 1.8), que cursaban desde $1^{\circ}$ de Educación Secundaria Obligatoria (ESO) hasta $2^{\circ}$ de Bachillerato en diferentes centros públicos (6) y privados concertados (3) de la Comunidad Autónoma de Galicia. Los porcentajes de alumnos de cada curso fueron similares (en torno al 16\%). Los centros fueron seleccionados de forma aleatoria entre el total de colegios de esta Comunidad.

Las pruebas se administraron en tres momentos diferentes: en octubre, los alumnos contestaron a la escala motivacional; en febrero cumplimentaron las escalas emocionales; a finales de junio, los profesores comunicaron la nota final en la asignatura de Lengua. El tiempo necesario para contestar a las dos escalas no superó los 15 minutos. Antes de la aplicación de los cuestionarios se solicitaron los correspondientes permisos a la dirección de los centros educativos y a los padres, en caso necesario. Los alumnos cumplimentaron los cuestionarios en el aula, dentro del horario de docencia, y ninguno se negó a contestarlos. Su aplicación en cada clase fue realizada por alguno de sus profesores. Previamente, éstos les explicaron el objetivo de la investigación y la conveniencia de sinceridad en las respuestas, ofreciéndoles garantías de anonimato y confidencialidad de las mismas. Para ello, se proporcionó a cada alumno un código, que mantuvo e incorporó a cada cuestionario en los diferentes momentos de la evaluación.

\section{Instrumentos}

\section{Motivación autodeterminada}

Para evaluarla, se aplicó la escala Perceived Locus of Causality (PLOC) de Goudas, Biddle y Fox (1994). Se adaptó su versión castellana (Moreno Murcia, González-Cutre y Chillón, 2009) refiriéndola a la asignatura de Lengua. Los alumnos estimaron en qué medida cada una de las razones para participar en la clase de Lengua española se correspondía con lo que les ocurría a ellos. Contestaron a 20 afirmaciones, cuatro para cada una de las siguientes modalidades de motivación: amotivación $(\alpha=.80)$ ("Realmente siento que estoy perdiendo mi tiempo en clase de Lengua"); regulación externa ( $\alpha=.85)$ ("Porque eso es lo que se supone que debo hacer"); regulación introyectada $(\alpha=.84$ ) ("Porque me sentiría mal conmigo mismo si no lo hiciera"); regulación identificada $(\alpha=.80)$ ("Porque es importante para mí hacerlo bien en la asignatura de Lengua"); y motivación intrínseca ( $\alpha=$ .73) ("Por la satisfacción que siento mientras aprendo cosas nuevas en esta materia"). Para cada ítem, los alumnos eligieron un valor entre 1 (en total desacuerdo) y 7 (totalmente de acuerdo). Se llevó a cabo un análisis factorial confirmatorio de esta escala, y los resultados indicaron que el modelo de cinco factores se ajustó bien a los datos de la muestra: $\chi^{2}(160, N=$ $642)=281.8, p<.001 ; \chi^{2} /$ g.l. $=1.76 ; \mathrm{AGFI}=.94 ; \mathrm{CFI}=$ .97 ; RMSEA $=.034$; SRMR $=.041$.

Como reconocen Goudas et al. (1994), el PLOC es una adaptación del Academic Self Regulation Questionnaire (SRQ-A) de Ryan y Connell (1989), un cuestionario extensamente utilizado con alumnos de secundaria. Por su parte, el PLOC se ha aplicado, sobre todo en secundaria, para evaluar la autodeterminación en la asignatura de educación física (Goudas et al. 1994; Moreno Murcia et al. 2009; Ntoumanis, 2002; Wang, Hagger y Liu, 2009; Wang y Liu, 2007) y en la realización de trabajos extensos (Liu et al. 2009). En general, reveló adecuados índices de fiabilidad y de ajuste a los datos en muestras de diferentes países.

\section{Emociones académicas}

Para su evaluación se aplicaron dos Escalas de Emociones Relacionadas con las Clases (Class-Related Emotion Scales) tomadas del Cuestionario de Emociones de Logro (Achievement Emotions Questionnaire-AEQ) de Pekrun, Goetz y Perry (2005). Se evaluaron dos emociones relacionadas con las clases de Lengua española, utilizando ocho ítems para cada una: disfrute $(\alpha=.90)$ ("Es emocionante que pueda estar en clase durante horas escuchando al profesor/a") y aburrimiento $(\alpha=.84)$ ("Miro el reloj a menudo porque el tiempo se me hace interminable"). Se solicitó a los estudiantes que describiesen cómo se sentían habitualmente antes de asistir a cla- 
ses de Lengua, durante las clases o después de las clases. Los alumnos estimaron su experiencia emocional en una escala con valores entre 1 (en total desacuerdo) y 5 (totalmente de acuerdo). Un análisis factorial confirmatorio indicó que el modelo de dos factores se ajustó adecuadamente a los datos de la muestra: $\chi^{2}(103, N=642)=289.1, p<.001 ; \chi^{2} /$ g.l. $=2.81$; $\mathrm{AGFI}=.94$; CFI $=.98 ; \mathrm{RMSEA}=.053$; $\mathrm{SRMR}=.023$.

Estas o similares escalas del AEQ han sido extensamente utilizadas, especialmente para evaluar las emociones en clase de diferentes materias como matemáticas, latín, inglés, alemán, física o psicología (Daniels et al., 2009; Dettmers et al., 2011; Frenzel et al., 2007, 2009; Goetz et al., 2006, 2007, 2008; Pekrun et al., 2010). Los coeficientes de fiabilidad confirman la adecuación de esta escala (con valores de $\alpha$ entre .84 y .94); también se ha constatado su validez estructural e interna (Pekrun et al., 2011).

La versión española de las Escalas de Emociones Relacionadas con las Clases se elaboró de acuerdo con el procedimiento habitual de traducción transcultural de escalas en tres fases. Primero, las escalas fueron traducidas del inglés al español, siguiendo los pasos sugeridos por el procedimiento de traducción en paralelo o traducción inversa (Brislin, 1986). A continuación un equipo seleccionó los ítems que habían mantenido el significado original y preparó las instrucciones y el formato de cada escala. Finalmente, se aplicó la versión española de las escalas a 12 estudiantes de secundaria para valorar la claridad y adecuación en la formulación de los ítems.

Rendimiento académico. Los profesores tutores de cada alumno comunicaron la nota final en Lengua obtenida por cada estudiante. Las puntuaciones oscilaron entre 1 y 10.

\section{Análisis de datos}

En primer lugar, se calcularon los coeficientes de fiabilidad (alfa de Cronbach), los estadísticos descriptivos y las correlaciones entre variables utilizando el paquete estadístico SPSS.15.

Luego, se realizó un análisis factorial confirmatorio para corroborar la idoneidad del modelo de medida, utilizando el programa AMOS.7 (Arbuckle, 2006). Finalmente, se realizó un análisis de ecuaciones estructurales, para contrastar el modelo de la Figura 1. En ambos análisis, como índices de ajuste presentamos los siguientes (Byrne, 2001): el indicador $\chi^{2}$, la medida más tradicional aunque muy sensible al tamaño de la muestra; el cociente $\chi^{2} /$ g.l., que se considera aceptable cuando sus valores son inferiores a 5 ; el índice de bondad de ajuste corregido (AGFI) y el índice de ajuste comparado (CFI), debiendo alcanzar ambos la puntuación de .90 o superior; el error cuadrático medio de aproximación (RMSEA) y la raíz del residuo estandarizado cuadrático medio (SRMR), considerándose valores razonables los incluidos entre $.08 \mathrm{y}$ .05 o menores.

Para estudiar las relaciones entre motivación y emoción se han utilizado con frecuencia los modelos de ecuaciones estructurales, pues su aplicación comporta un buen número de ventajas respecto a otros métodos (Tomarken y Waller 2005): permiten analizar datos que no cumplen la normalidad estadística; posibilitan la introducción de conocimiento teórico en la especificación del modelo antes de su estimación; ponen a prueba el fenómeno a estudiar incorporando múltiples variables endógenas y exógenas; utilizan variables latentes, cada una de ellas evaluada mediante múltiples indicadores; y tienen en cuenta el papel de las variables mediadoras y no sólo los efectos directos de unas variables sobre otras.

Investigaciones recientes han utilizado modelos de ecuaciones estructurales (o análisis similares) para estudiar los nexos entre emociones, motivación y rendimiento. Así, Daniels et al. (2009) comprobaron que disfrute, aburrimiento y ansiedad mediaron las relaciones entre metas de logro y notas. Análogamente, Pekrun et al. (2010) constataron que el aburrimiento fue un mediador entre la motivación (control percibido y valor de la tarea) y las notas. Asimismo, Dettmers et al. (2011) encontraron que las emociones desagradables y el esfuerzo mediaron parcialmente la relación entre motivación (expectativa y valor) y rendimiento. De acuerdo con Núñez et al. (2011), la inteligencia emocional percibida medió las relaciones entre orientación motivacional a la tarea y el bienestar psicológico en deportistas de diferentes edades; de modo similar, el índice de autodeterminación medió la influencia que ejercen el clima motivacional y las metas de logro sobre el flow disposicional en deportistas adolescentes (Moreno, Cervelló y González, 2010).

En el análisis de la mediación, el procedimiento más utilizado consta de cuatro etapas (Holmbeck, 1997; Wu y Zumbo, 2008). En la etapa 1 se muestra si la variable independiente predice la variable dependiente; esta fase establece si entre ambas existe un efecto directo significativo que, luego, puede ser mediado por otras variables. La etapa 2 analiza si la variable independiente se relaciona con el mediador; en esta fase el mediador es tratado como una variable dependiente. En la etapa 3 se muestra si el mediador afecta a la variable dependiente, si ésta es predicha conjuntamente por la variable independiente y por el mediador. Finalmente, en la etapa 4 se analizan los efectos directos e indirectos obtenidos en la etapa 3; existirá mediación si los efectos indirectos son significativos, lo que hará disminuir en la misma proporción los efectos directos.

\section{Resultados}

\section{Análisis preliminares}

En la Tabla 1 podemos ver los estadísticos descriptivos y los coeficientes de correlación entre las variables. En ella constatamos que la media de la amotivación y de la regulación externa son menores que las del resto de modalidades de motivación. Además, la puntuación en disfrute superó a la del aburrimiento. 
Tabla 1. Estadísticos descriptivos y correlaciones entre variables.

\begin{tabular}{lllllllllll}
\hline Variables & Rango $M$ & $D T$ & 1 & 2 & 3 & 4 & 5 & 6 & 7 \\
\hline 1. Amotivación & $1-7$ & 3.10 & 1.06 & - & & & & & & \\
2. R. Externa & $1-7$ & 3.01 & 1.18 & .35 & - & & & & & \\
3. R. Introyectada & $1-7$ & 3.97 & 1.10 & -.12 & -.16 & - & & & & \\
4. R. Identificada & $1-7$ & 4.00 & .98 & -.16 & -.27 & .14 & - & & & \\
5. M. Intrínseca & $1-7$ & 4.11 & .83 & -.24 & -.13 & .09 & .23 & - & & \\
6. Aburrimiento & $1-5$ & 2.16 & .85 & .38 & .34 & -.16 & -.28 & -.31 & - & \\
7. Disfrute & $1-5$ & 3.03 & 1.02 & -.33 & -.41 & .19 & .29 & .29 & -.50 & - \\
8. Rendimiento & $1-10$ & 6.44 & 1.92 & -.42 & -.43 & .29 & .39 & .36 & -.50 & .48 \\
\hline Nota. $|r| \geq .09=p<.05 ;$ & $|r| \geq .11=p<.01$. & & & & & &
\end{tabular}

En cuanto a las correlaciones, éstas agrupan a las diferentes formas de motivación en dos bloques: uno con las menos autodeterminadas, amotivación y regulación externa; otro con las más autodeterminadas, motivación intrínseca, regulación identificada e introyectada. Dentro de cada grupo, las correlaciones son positivas, y negativas las que las unen al otro grupo. Las más autodeterminadas correlacionan positivamente con el disfrute y el rendimiento, y negativamente con el aburrimiento; en las menos autodeterminadas encontramos un patrón inverso de correlaciones. El nexo entre disfrute y aburrimiento es negativo.

\section{Modelo de medida}

Como se ha dicho, cada emoción se evaluó mediante ocho ítems y cada modalidad de motivación mediante cuatro indicadores, lo que suma un total de 36 variables observadas. Con el fin de simplificar el modelo, como recomiendan Bentler y Chou (1987) y Byrne (2001), para los análisis factoriales y el de ecuaciones estructurales se agruparon los ítems de las escalas emocionales. Entre los múltiples métodos de agrupamiento se eligió uno, sugerido por Little, Cinningham, Shahar y Widaman (2002) y utilizado con estas mismas escalas por Daniels et al. (2009) y Pekrun et al. (2010): calcular la media de dos ítems correlacionados entre sí y con significados similares. Para cada emoción, este agrupamiento dio origen a cuatro parcels cuyas medias se utilizaron como indicadores en los diferentes análisis. Los indicadores de cada tipo de motivación fueron sus cuatro ítems.

Para corroborar la idoneidad de las escalas aplicadas, se realizó un análisis factorial confirmatorio para todas las variables, utilizando el programa AMOS.7. En todos los indicadores se obtuvieron índices de asimetría y curtosis menores que $|1.96|$, por lo que puede asumirse la normalidad univariada (Arbuckle, 2006; Byrne, 2001). En ningún caso se encontraron observaciones atípicas multivariadas (outliers). Sin embargo, el coeficiente de curtosis multivariada de Mardia (23.6) superó el valor crítico (critical ratio, c.r. $=7.3$ ). Para verificar si esta no-normalidad influía en los distintos estimadores, se realizaron dos tipos de análisis (Arbuckle, 2006; Byrne, 2001): uno con la muestra original, mediante el método de máxima verosimilitud; otro con 500 muestras aleatorias obtenidas a partir de la original por el procedimiento de remuestreo (bootstrap), también mediante el método de máxima verosimilitud; además, se marcó un intervalo del 95\% para evaluar las diferencias entre ambos análisis. Las divergencias entre los estimadores calculados por ambos métodos (sesgos) fueron mínimas, diferenciándose sólo en el tercer decimal; además, ninguno de los intervalos de confianza corregidos respecto al sesgo incluyó el cero; de todo ello inferimos que la no-normalidad multivariada no afectó a la precisión de los estimadores (Byrne, 2001). Por este motivo, a continuación se presentan los resultados de los análisis realizados sobre la muestra original. No se efectuaron reespecificaciones del modelo inicial.

Los indicadores revelan un adecuado ajuste del modelo de medida a los datos: $\chi^{2}(328, N=642)=574.1, p<.001$; $\chi^{2} /$ g.l. $=1.75 ;$ AGFI $=.92 ;$ CFI $=.97 ;$ RMSEA $=.034 ;$ $\mathrm{SRMR}=.042$. Además, todas las cargas factoriales estandarizadas fueron significativas $(\beta>.60 ; p<.01)$. Esto nos indicó que el modelo de medida fue adecuado.

\section{Modelo estructural}

A continuación se llevaron a cabo tres análisis de ecuaciones estructurales, según las etapas a seguir para evaluar la mediación entre variables (Holmbeck, 1997; Wu y Zumbo, 2008). El coeficiente de curtosis multivariada de Mardia también aquí superó el valor crítico, con un índice de 21.07 $(c . r .=6.29)$ para el modelo mediacional final. Por este motivo, se realizaron los dos tipos de análisis aplicados en el modelo de medida: de la muestra original, mediante el método de máxima verosimilitud; y de 500 muestras obtenidas por remuestreo, también mediante el método de máxima verosimilitud; se fijó un intervalo del 95\% para evaluar el sesgo entre ambos análisis. Puesto que no existieron diferencias significativas entre los dos análisis, se presentarán los estimadores obtenidos con la muestra original. Como índices de ajuste se eligieron los mismos que en el análisis factorial. No se realizaron re-especificaciones del modelo inicial.

Analizando la relación entre las modalidades de motivación y el rendimiento (etapa 1), encontramos un adecuado ajuste del modelo a los datos, $\chi^{2}(174, N=642)=323.2, p$ $<.001 ; \chi^{2} /$ g.l. $=1.86 ; \mathrm{AGFI}=.94 ; \mathrm{CFI}=.97 ; \mathrm{RMSEA}=$ .037 ; SRMR $=.041$. La amotivación se asoció negativamente al rendimiento $(\beta=-.23, p<.01)$ al igual que la regulación externa $(\beta=-.21, p<.01)$; en cambio, se relacionaron positivamente con el rendimiento la regulación introyectada $(\beta=$ $.18, p<.01)$, la regulación identificada $(\beta=.23, p<.01)$ y la motivación intrínseca $(\beta=.24, p<.01)$. Conjuntamente, las cinco modalidades de autorregulación explicaron el $45 \%$ de la varianza en rendimiento académico en Lengua.

Examinando los nexos entre las diferentes modalidades de motivación y las emociones (etapa 2), los índices revelan un adecuado ajuste del modelo a los datos, $\chi^{2}(329, N=642)$ $=628.8, p<.001 ; \chi^{2} /$ g.l. $=1.91 ; \mathrm{AGFI}=.92 ; \mathrm{CFI}=.96$; RMSEA $=.038$; SRMR $=.049$. Los diferentes tipos de motivación predijeron significativamente los dos mediadores: la amotivación y la regulación externa fueron predictores positivos del aburrimiento $(\beta=.27, p<.01$ y $\beta=.20, p<.01$, 
respectivamente) y predictores negativos del disfrute $(\beta=$ $.15, p<.01$ y $\beta=-.31, p<.01)$; relaciones inversas las encontramos para la regulación identificada y la motivación intrínseca, que predijeron negativamente el aburrimiento $(\beta=$ $.13, p<.01$ у $\beta=-.26, p<.01)$ y positivamente el disfrute $(\beta=.13, p<.01$ y $\beta=.24, p<.01)$. La regulación introyectada fue un predictor negativo no significativo del aburri- miento $(\beta=-.07, p>.08)$ y un predictor positivo poco intenso del disfrute $(\beta=.09, p<.05)$.

El modelo mediacional final (etapa 3), representado en la Figura 2, mostró buenos niveles de ajuste a los datos, $\chi^{2}$ $(350, N=642)=695.7, p<.001 ; \chi^{2} /$ g.l. $=1.98 ;$ AGFI $=$ .91 ; CFI $=.96$; RMSEA $=.039$; SRMR $=.049$. Las saturaciones de los indicadores sobre su respectiva variable latente superaron el valor de $.60(p<.01)$.

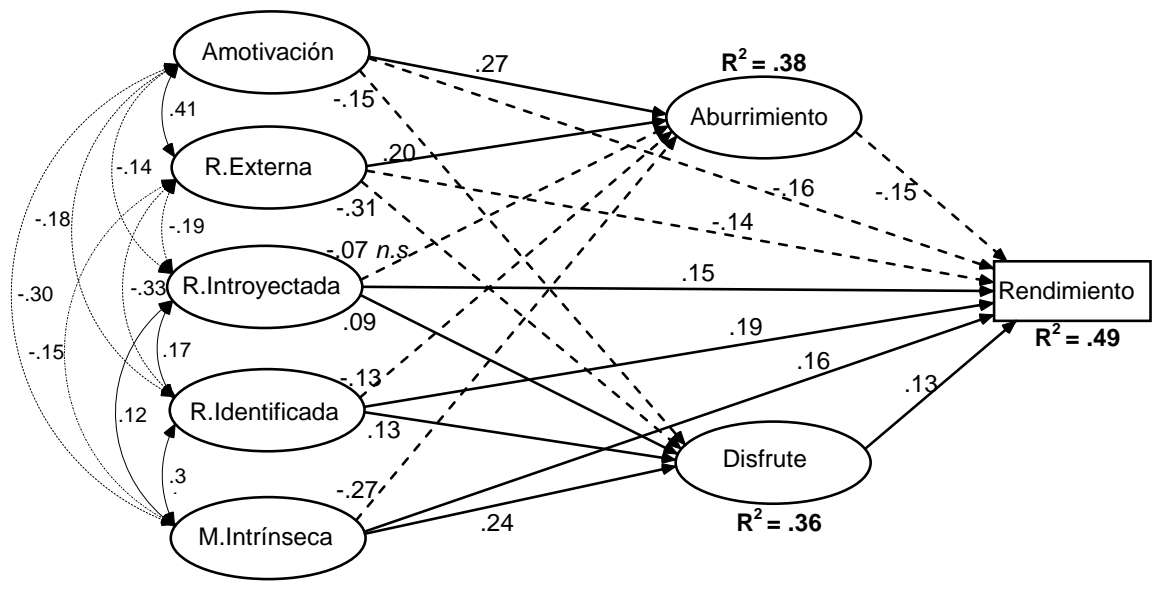

Figura 2. Modelo estructural de relaciones entre variables (parámetros estandarizados). n.s. = no significativo; los demás parámetros son significativos $(p<.05)$. Las líneas discontinuas indican relaciones negativas.

Seguidamente, evaluamos si existía la invarianza de género en este modelo mediacional aplicando el test de diferencia de chi cuadrado (Byrne 2001). Para ello, se impuso la restricción de que todos los coeficientes de regresión fueran equivalentes en las dos muestras. Los resultados evidenciaron que no existían diferencias significativas según el género, lo que nos llevó a asumir la equivalencia en las relaciones estructurales para los dos géneros.

En la Figura 2 vemos que la regulación externa y, especialmente, la desmotivación fueron predictores positivos del aburrimiento, mientras que la regulación identificada y la motivación intrínseca lo predijeron negativamente; la relación entre regulación introyectada y aburrimiento, aunque negativa, no fue significativa; las modalidades de autodeterminación explicaron el $38 \%$ de la varianza en aburrimiento. La regulación introyectada, la identificada y la motivación intrínseca fueron predictores positivos del disfrute en clases, mientras que la amotivación y la regulación externa lo predijeron negativamente; las diversas formas de motivación explicaron un $36 \%$ de la varianza en disfrute. Los efectos directos sobre el rendimiento fueron significativos para todas las modalidades de motivación autodeterminada; también lo fueron para el disfrute y el aburrimiento. Conjuntamente, las variables evaluadas explicaron un $49 \%$ de la varianza en rendimiento académico en Lengua.

Finalmente (etapa 4), comparamos los efectos directos (de las modalidades de autodeterminación sobre el rendimiento) obtenidos en la etapa 1 con los efectos directos de la etapa 3. Según se recoge en la Tabla 2 , al incluir los mediadores en el modelo se redujeron los efectos directos de las diferentes modalidades de motivación sobre el rendimiento, a pesar de lo cual esos efectos directos se mantuvieron significativos. Además, la suma de los efectos indirectos, mediados a través del aburrimiento y el disfrute, fue significativa para todos los tipos de motivación autodeterminada. Todo ello confirma la existencia de mediación entre las formas de autodeterminación y el rendimiento académico a través de las dos emociones evaluadas, aburrimiento y disfrute en clase.

Tabla 2. Efectos las modalidades de motivación sobre el rendimiento.

\begin{tabular}{lccc}
$\begin{array}{l}\text { Modalidad de } \\
\text { motivación }\end{array}$ & $\begin{array}{c}\text { Efecto } \\
\text { Directo }\end{array}$ & $\begin{array}{c}\text { Suma de efectos } \\
\text { indirectos }(*)\end{array}$ & $\begin{array}{c}\text { Efecto } \\
\text { Total }(*)\end{array}$ \\
\hline Amotivación & $-.160(p<.01)$ & $-.068(p<.003)$ & $-.228(p<.004)$ \\
R. Externa & $-.140(p<.01)$ & $-.073(p<.004)$ & $-.213(p<.003)$ \\
R. Introyectada & $.151(p<.01)$ & $.024(p<.028)$ & $.175(p<.005)$ \\
R. Identificada & $.188(p<.01)$ & $.040(p<.008)$ & $.228(p<.006)$ \\
M. Intrínseca & $.164(p<.01)$ & $.078(p<.002)$ & $.242(p<.004)$ \\
\hline (*) La probabilidad asociada a cada suma de efectos indirectos y cada efecto \\
total se obtuvo mediante el Bias-corrected confidence interval bootstrap \\
test del AMOS.7 (nivel de confianza $=95 \%$; muestras = 500).
\end{tabular}

\section{Discusión}

El objetivo del presente trabajo fue analizar, en una muestra de estudiantes de secundaria, los nexos entre distintas modalidades de motivación autodeterminada, aburrimiento, dis- 
frute y rendimiento académico. Son pocos los trabajos que han relacionado estas variables, y menos todavía los que las han referido a la asignatura de Lengua española, la única materia obligatoria para todos los estudiantes de secundaria y bachillerato.

Después de calcular los coeficientes de fiabilidad, los estadísticos descriptivos y las correlaciones entre variables, se determinó la idoneidad del modelo de medida y se llevó a cabo un análisis de ecuaciones estructurales. Los dos instrumentos de evaluación utilizados evidenciaron niveles de ajuste aceptables y adecuada fiabilidad. Asimismo, los distintos índices revelaron un buen ajuste entre el modelo estructural hipotetizado y los datos de la muestra.

\section{Relaciones entre variables}

Según lo esperado, la ausencia de razones para estudiar Lengua y la escasa valoración de esta asignatura (características de la amotivación) hicieron más probable que el alumno se aburriera en clase; también favoreció el aburrimiento la percepción de estudiar por obligación o porque no existe otra alternativa (regulación externa); en cambio, representaron una protección frente al aburrimiento aquellas razones que resaltan el interés de la asignatura por sí misma o la importancia para el futuro del alumno. Estas razones identificadas e intrínsecas fueron las que más intensamente se asociaron con el disfrute en las clases; en cambio, la desmotivación y la regulación externa dificultaron ese disfrute. En estos casos quedaron claros los nexos entre motivación y emociones, lo que no ocurrió de forma tan evidente con la regulación introyectada: aunque el signo de sus efectos fue el esperado, la intensidad de los mismos no fue muy elevada, situándose esta forma de regulación a medio camino entre la desmotivación y la motivación intrínseca. En general, los datos son coherentes con los de investigaciones previas con estudiantes de secundaria en clase de educación física (Ntoumanis, 2002; Sas-Nowosielski, 2008; Wang y Liu, 2007) o durante la elaboración de trabajos académicos extensos (Liu et al., 2009). En el presente estudio, la motivación intrínseca se reveló como la modalidad de motivación más adaptativa, siendo la amotivación la menos apropiada; entre ambas se situaron las demás formas de autodeterminación. En cuanto a los datos referidos a la regulación introyectada, coinciden con la mayoría de investigaciones previas que no encontraron nexos unívocos entre esta forma de motivación y las emociones. Esta ambigüedad podría explicarse si consideramos la existencia de dos tipos de regulación introyectada, de aproximación y de evitación, tal como proponen Assor et al. (2009): la primera (por la que el alumno intenta lograr o mantener elevados sentimientos de autoestima, auto-valor, orgullo y aprobación social) podría explicar la capacidad de la regulación introyectada para predecir positivamente el disfrute; la regulación introyectada de evitación (por la que el alumno intenta evitar sentimientos de bajo auto-valor, de vergüenza o de culpabilidad) podría explicar la escasa capa- cidad de esta forma de motivación para proteger al estudiante frente al aburrimiento.

Conjuntamente, las diferentes modalidades de motivación autodeterminada, el aburrimiento y el disfrute, explicaron un elevado porcentaje de la varianza en la nota final obtenida por los alumnos en Lengua. La capacidad de las modalidades de autodeterminación para predecir el rendimiento también puede considerarse gradual: la amotivación fue el predictor negativo más intenso, seguido de la regulación externa; la motivación intrínseca, seguida de la regulación identificada, resultó ser el predictor positivo más intenso. Estos resultados confirman y amplían la investigación previa referida a las diferentes modalidades de motivación (Boiché et al., 2008; Liu et al., 2009; Ntoumanis, 2002; Ryan y Deci, 2009), al aburrimiento (Acee et al., 2010; Nett et al., 2010, 2011; Pekrun et al., 2010) y al disfrute (Ainley y Ainley, 2011; Frenzel et al., 2007, 2009; Goetz et al., 2006, 2007, 2008).

De especial interés podemos considerar los resultados referidos a las relaciones indirectas entre motivación autodeterminada y rendimiento. El modelo de mediación propuesto, en el que aburrimiento y disfrute en clase mediaban las intensas relaciones entre formas de autodeterminación y rendimiento, se confirmó en este estudio. El efecto directo positivo de la motivación intrínseca ( $\mathrm{y}$, en menor medida, de la regulación identificada y la introyectada) sobre el rendimiento se explicó en parte porque cada una de ellas incrementó el disfrute, asociado a un mayor rendimiento, y protegió frente al aburrimiento, un potente predictor de resultados deficientes. De manera análoga, los fuertes efectos directos negativos de la amotivación (y, más moderados, de la regulación externa) sobre el rendimiento se explicaron parcialmente porque ambas favorecieron el aburrimiento y dificultaron el disfrute; niveles elevados de aburrimiento y bajos de disfrute en clases anticipan un rendimiento deficiente. Para todas las modalidades de motivación, los efectos indirectos fueron significativos, aunque también lo fueron los efectos directos para todas ellas. Estos resultados son coherentes con algunos estudios en los que emociones específicas (disfrute, aburrimiento o ansiedad) mediaron los nexos positivos o negativos entre diferentes constructos motivacionales (metas de logro, control percibido, valor de la tarea o expectativa) y rendimiento académico (Daniels et al., 2009; Dettmers et al., 2011; Pekrun et al., 2010).

\section{Implicaciones educativas}

Los resultados de este estudio, junto a la investigación previa, prestan apoyo al continuo motivacional propuesto por los autores de la teoría de la autodeterminación, sustituyendo a la dicotomía de motivación extrínseca-intrínseca. Esta nueva concepción ofrece el marco adecuado para la puesta en práctica de programas de intervención en los que profesores y padres facilitan en sus estudiantes/hijos el proceso de internalización, mediante el cual las regulaciones externas al sujeto se transforman en valores asumidos por él. Estas conductas se agrupan en torno a tres ejes: apoyo a la 
autonomía en el proceso de aprendizaje del alumno/hijo, implicación activa en su educación, y presentación de un entorno estructurado para el aprendizaje en clase/casa. Estas estrategias pueden ser puestas en práctica tanto por los profesores (Jang, Reeve y Deci, 2010; Ryan y Deci, 2009) como por los padres (Grolnick, Friendly y Bellas, 2009; Roth et al., 2009) y tienen múltiples efectos beneficiosos sobre la motivación, las emociones, el compromiso académico, la persistencia y el rendimiento de los estudiantes, como señala Reeve (2009) en una reciente revisión.

Además, nuestros resultados referidos a las emociones apoyan diversas sugerencias encaminadas a incrementar el disfrute y el interés (Ainley y Ainley, 2011; Frenzel et al., 2009; Goetz et al., 2006, 2008) y a evitar o reducir el aburrimiento en clase (Acee et al., 2010; Nett et al., 2010, 2011; Pekrun et al., 2010), asumiendo que ambas emociones pueden deberse a razones individuales y contextuales. Algunas propuestas dirigen sus actividades al componente personal: fomentar la internalización, como ya se ha indicado; aumentar el valor subjetivo de las tareas, reconociendo su importancia para la consecución de metas personales futuras; atribuir los fracasos a causas controlables, como la falta de adecuadas estrategias o el esfuerzo insuficiente; puesto que las actividades escolares aburridas pocas veces pueden evitarse, adquirir adecuadas estrategias de afrontamiento, como reflexionar sobre la utilidad de una actividad aburrida. El objetivo de otras actuaciones es el componente contextual de las emociones: proponer en clase actividades de diferentes niveles de dificultad, pues provocan aburrimiento tanto las muy fáciles como las demasiado difíciles; despertar el interés de los estudiantes (ej. eligiendo distintos temas o utilizando materiales diversos), mantenerlo (ej. especificando la relevancia del tema o proponiendo tareas variadas) y manifestarlo (ej. introduciendo las tareas de forma positiva o proyectando entusiasmo en lo que se comunica).

\section{Limitaciones y futuras investigaciones}

A pesar de que el modelo propuesto resultó útil para explicar las relaciones entre las variables estudiadas, señalamos algunas limitaciones que pueden servir de guía a trabajos fu-

\section{Referencias}

Acee, T., Kim, H., Kim, H., Kim, J., Chu, H., Kim, M., Cho, Y., Wicker, F. y The Boredom Research Group (2010). Academic boredom in underand over-challenging situations. Contemporary Educational Psychology, 35, $17-27$.

Ainley, M. y Ainley, J. (2011). Student engagement with science in early adolescence: The contribution of enjoyment to students' continuing interest in learning about science. Contemporary Educational Psychology, 36, 4-12.

Arbuckle, J. (2006). AMOS 7.0. User's Guide. Chicago, IL: Smallwaters Corporation.

Assor, A., Vansteenkiste, M. y Kaplan, A. (2009). Identified versus introjected approach and introjected avoidance motivation in school and in sports: The limited benefits of self-worth strivings. Journal of Educational Psychology, 101, 482-497.

Bentler, P. y Chou, P. (1987). Practical issues in structural modelling. Sociological Methods \& Research, 16, 78-117. turos. Aunque la regulación integrada se incluye dentro de la teoría de la autodeterminación, en nuestro estudio (como en la totalidad de los citados) no se ha evaluado; sin embargo, como reconocen González-Cutre et al. (2010), es una modalidad de motivación autodeterminada muy relevante para explicar la implicación de los sujetos en una tarea concreta, por lo que sería de gran interés estudiarla en el futuro. Tampoco se han analizado los condicionantes de la autodeterminación, por lo que sería de gran ayuda incorporar al modelo ciertas percepciones de los alumnos, como la del apoyo a la autonomía en el aula o en casa (Jang et al., 2010; Roth et al., 2009), que condicionan sus formas de motivación y sus emociones. También resultaría muy revelador abordar otras consecuencias de la motivación y las emociones, como la utilización de estrategias de aprendizaje (Rinaudo, Chiecher y Donolo, 2003) o el compromiso de los estudiantes con sus tareas escolares (Ryan y Deci, 2009) contrapuesto a la desafección, la indiferencia y el abandono del colegio. Finalmente, como sugiere el último informe PISA (Ministerio de Educación, 2010), consideramos muy importante investigar los efectos del rendimiento sobre la motivación y las emociones, y de éstas sobre aquélla, para obtener un dibujo más completo de las relaciones entre todas estas variables.

Para concluir, como resultados más relevantes de este estudio apuntamos los siguientes: la idoneidad de las escalas utilizadas para evaluar la motivación autodeterminada y las emociones académicas en una materia en secundaria; la relativa gradación en los nexos de las diferentes modalidades de autodeterminación con las emociones y el rendimiento; la capacidad del disfrute y el aburrimiento en clases para predecir el rendimiento académico; y el papel de estas dos emociones académicas como mediadores entre la motivación autodeterminada y el rendimiento. Este último hallazgo del estudio representa su principal aportación a la investigación previa, pues ayuda a entender algo más la intensa relación entre motivación autodeterminada y rendimiento: los alumnos con razones más autodeterminadas para asistir a clase de Lengua obtuvieron mejores resultados en esta materia, entre otras razones, porque disfrutaron más de las clases y se aburrieron menos en ellas, factores ambos que favorecen la implicación en las tareas, el aprendizaje y el rendimiento.

Brislin, R. (1986). The wording and translation of research instruments. En W. Lonner y J. Berry (Eds.), Field methods in cross-cultural research (pp. 137164). Beverly Hills, CA: Sage.

Boiché, J., Sarrazin, P., Grouzet, F., Pelletier, L. y Chanal, J. (2008). Students' motivational profiles and outcomes in physical education: A selfdetermination perspective. Journal of Educational Psychology, 100, 688-701.

Byrne, B. (2001). Structural equation modeling with AMOS. Basic concepts, applications, and programming. Mahwah, NJ: LEA.

Daniels, L., Stupnisky, R., Pekrun, R., Havnes, T., Perry, R. y Newall, N. (2009). A longitudinal analysis of achievement goals: From affective antecedents to emotional effects and achievement outcomes. Journal of Educational Psychology, 101, 948-963.

Daschmann, E., Goetz, T. y Stupnisky, R. (2011). Testing the predictors of boredom at school: Development and validation of the precursors to boredom scales. British Journal of Educational Psychology, 81, 421-440. 
Deci, E. y Ryan, R. (2000). The "what" and "why" of goal pursuits: Human needs and the self-determination of behavior. Psychological Inquiry, 11, 227-268.

Dettmers, S., Trautwein, U., Lüdke, O., Goetz, T., Frenzel, A. y Pekrun, R. (2011). Students' emotions during homework in mathematics: Testing a theoretical model of antecedents and achievement outcomes. Contemporary Educational Psychology, 36, 25-35.

Fernández, M., Mena, L. y Riviere, J. (2010). Fracaso y abandono escolar en España. Barcelona: Fundación la Caixa.

Frenzel, A., Goetz, T., Lüdtke, O., Pekrun, R. y Sutton, R. (2009). Emotional transmission in the classroom: Exploring the relationship between teacher and student enjoyment. Journal of Educational Psychology, 101, 705-716.

Frenzel, A., Pekrun, R. y Goetz, T. (2007). Perceived learning environment and students' emotional experiences: A multilevel analysis of mathematics classroom. Learning and Instruction, 17, 478-493.

Goetz, T., Frenzel, A., Hall, N. y Pekrun, R. (2008). Antecedents of academic emotions: Testing the internal/external frame of reference model for academic enjoyment. Contemporary Educational Psychology, 33, 9-33.

Goetz, T., Frenzel, A., Pekrun, R., Hall, N. y Lüdtke, O. (2007). Betweenand within-domain relations of students' academic emotions. Journal of Educational Psychology, 99, 715-733.

Goetz, T., Hall, N., Frenzel, A. y Pekrun, R. (2006). A hierarchical conceptualization of enjoyment in students. Learning and Instruction, 16, 323-338.

González-Cutre, D., Sicilia, A. y Fernández, A. (2010). Hacia una mayor comprensión de la motivación en el ejercicio físico: medición de la regulación integrada en el contexto español. Psicothema, 22, 841-847.

Goudas, M., Biddle, S. y Fox, K. (1994). Perceived locus of causality, goal orientations, and perceived competence in school physical education classes. British Journal of Educational Psychology, 64, 453-463.

Grolnick, W., Friendly, R. y Bellas, V. (2009). Parenting and children's motivation at school. In K. Wentzel y A. Wigfield (Eds.), Handbook of motivation at school (pp.279-300). New York: Routledge.

Holmbeck, G. (1997). Toward terminological, conceptual, and statistical clarity in the study of mediators and moderators: Examples from the child-clinical and pediatric psychology literatures. Journal of Consulting and Clinical Psychology, 65, 599-610.

Jang, H., Reeve, J. y Deci, E. (2010). Engaging students in learning activities: It is not autonomy support or structure but autonomy support and structure. Journal of Educational Psychology, 102, 588-600.

Little, T., Cinningham, W., Shahar, G. y Widaman, K. (2002). To parcel or not to parcel: Exploring the question, weighing the merits. Structural Equation Modeling, 9, 151-173.

Liu, W., Wang, C., Tan, O., Koh, C. y Ee, J. (2009). A self-determination approach to understanding students' motivation in Project work. Learning and Individual Differences, 19, 139-145.

Meyer, D. y Turner, J. (2007). Scaffolding emotions in classroom. En P. Schutz y R. Pekrun (Eds.), Emotion in education (pp. 243-258). San Diego: Academic Press.

Ministerio de Educación (2010). PIS A 2009. Programa para la Evaluación Internacional de los Alumnos OCDE. Informe Español. Madrid: Ministerio de Educación.

Moreno, J., Cervelló, E. y González, D. (2010). The achievement goal and self-determination theories as predictors of dispositional flow in young athletes. Anales de Psicologia, 26, 390-399.

Moreno Murcia, J., González-Cutre, D. y Chillón, M. (2009). Preliminary validation in Spanish of a scale designed to measure motivation in physical education classes: The Perceived Locus of Causality (PLOC) Scale. The Spanish Journal of Psychology, 12, 327-337.

Nett, U., Goetz, T. y Daniels, L. (2010). What to do when feeling bored? Students' strategies for coping with boredom. Learning and Individual Differences, 20, 626-638.

Nett, U., Goetz, T. y Hall, N. (2011). Coping with boredom: An experience sampling perspective. Contemporary Educational Psychology, 36, 49-59
Ntoumanis, N. (2002). Motivational clusters in a sample of British physical education classes. Psychology of Sport and Exercise, 3, 177-194.

Núñez, J. L., León, J., González, V. y Martín-Albo, J. (2011). Propuesta de un modelo explicativo del bienestar psicológico en el contexto deportivo. Revista de Psicología del Deporte, 20, 223-242.

Pekrun, R. (2006). The control-value theory of achievement emotions: Assumptions, corollaries, and implications for educational research and practice. Educational Psychology Review, 18, 315-341.

Pekrun, R. (2009). Emotions at school. En K. Wentzel y A. Wigfield (Eds.), Handbook of motivation at school (pp. 575-604). London: Routledge.

Pekrun, R., Frenzel, A., Goetz, T. y Perry, R. (2007). The control-value theory of achievement emotions: An integrative approach to emotions in education. En P. Schutz y R. Pekrun (Eds.), Emotion in education (pp. 13 36). San Diego: Academic Press.

Pekrun, R., Goetz, T., Daniels, L., Stupnisky, R. y Perry, R. (2010). Boredom in achievement settings: Exploring control-value antecedents and performance outcomes of a neglected emotion. Journal of Educational Psychology, 102, 531-549.

Pekrun, R., Goetz, T., Frenzel, A., Barchfeld, P. y Perry, R. (2011). Measuring emotions in students` learning and performance: The Achievement Emotions Questionnaire (AEQ). Contemporary Educational Psychology, 36, 36-48.

Pekrun, R., Goetz, T. y Perry, R. (2005). Achievement Emotions Questionnaire (AEQ)- user's Manual. University of Munich: Department of Psychology.

Reeve, J. (2009). Why teachers adopt a controlling motivating style toward students and how they can become more autonomy supportive. Educational Psychologist, 44, 159-175.

Rinaudo, C., Chiecher, A. y Donolo, D. (2003). Motivación y uso de estrategias en estudiantes universitarios. Su evaluación mediante el Motivated Strategies Learning Questionnaire. Anales de Psicología, 19, 107-119.

Roth, G., Assor, A., Niemiec, C., Ryan, R. y Deci, E. (2009). The emotional and academic consequences of parental conditional regard: Comparing conditional positive regard, conditional negative regard, and autonomy support as parenting practices. Developmental Psychology, 45, 1119-1142.

Ryan, R. y Connell, J. (1989). Perceived locus of causality and internalization: Examining reasons for acting in two domains. Journal of Personality and Social Psychology, 57, 749-761.

Ryan, R. y Deci, E. (2002). An overview of self-determination theory: An organismic dialectic perspective. En E. Deci y R. Ryan (Eds.), Handbook of self-determination research (pp. 3-33). Rochester: University of Rochester Press.

Ryan, R. y Deci, E. (2009). Promoting self-determined school engagement: Motivation, learning, and well-being. En K. Wentzel y A. Wigfield (Eds.), Handbook of motivation at school (pp. 171-195). New York: Routledge.

Sas-Nowosielski, K. (2008). Participation of youth in physical education from the perspective of self-determination theory. Human Movement, 9 , 134-141.

Tomarken, A. y Waller, N. (2005). Structural equation modeling: Strengths, limitations, and misconceptions. Annual Review of Clinical Psychology, 1 , $31-65$

Vallerand, R. (1997). Toward a hierarchical model of intrinsic and extrinsic motivation. En M. Zanna (Ed.), Advances in experimental social psychology (vol. 29, pp. 271-360). New York: Academic Press.

Wang, C., Hagger, M. y Liu, W. (2009). A cross-cultural validation of Perceived Locus of Causality Scale in physical education context. Research Quarterly for Exercise and Sport, 80, 313-325.

Wang, C. y Liu, W. (2007). Promoting enjoyment in girls' physical education: The impact of goals, beliefs, and self-determination. European Physical Education Review, 13, 145-164.

Wu, A. y Zumbo, B. (2008). Understanding and using mediators and moderators. Social Indicators Research, 87, 367-392.

(Artículo recibido: 19-9-2011, revisado: 27-03-2012, aceptado: 27-03-2012) 